\title{
Estudo retrospectivo dos pacientes portadores de melanoma cutâneo atendidos na Universidade Federal de São Paulo.
}

\section{Retrospective study of patients with cutaneous melanoma treated at the Federal University of São Paulo.}

Tácito Ferreira'; Ivan Dunshee de Abranches Oliveira Santos'; Andrea Fernandes Oliveira'; Lydia Masako Ferreira, TCBC-SP1

\author{
R E S U M O
}

\begin{abstract}
Objetivo: avaliar as características dos pacientes portadores de melanoma cutâneo atendidos no Hospital São Paulo UNIFESP. Métodos: estudo retrospectivo de 184 casos de melanoma cutâneo. Foram analisadas as informações sobre sexo, idade, características do tumor, características histológicas e estadiamento. Resultados: a média de idade ao diagnóstico foi de 58,7 anos, com distribuição etária homogênea entre os sexos e predominância em indivíduos brancos $(70,6 \%)$. Observou-se acometimento predominante de tronco, em homens $(36,7 \%)$, e de membros inferiores, em mulheres (42\%). A exposição solar, com queimaduras, foi mais comum entre homens (31,2\%) do que entre mulheres $(23,5 \%)$. Houve aumento de aproximadamente três vezes no acometimento linfonodal quando o índice mitótico subia de zero $(11,9 \%)$ para uma ou mais mitoses por campo $(36,2 \%)$, e aumento progressivo do acometimento linfonodal e de desfechos ruins quanto maior a espessura de Breslow: 10,2\% quando menor do que $1 \mathrm{~mm}$ e $59,2 \%$ quando maior do que $4 \mathrm{~mm}$. Conclusão: as características dos pacientes portadores de melanoma cutâneo atendidos no Hospital São Paulo são semelhantes às encontradas na literatura.
\end{abstract}

Descritores: Melanoma. Neoplasias Cutâneas. Mitose. Fatores de Risco. Melanoma/epidemiologia.

\section{INTRODUÇÃO}

$\mathrm{O}$ câncer de pele é a forma mais comum de câncer, representando cerca de 40 a 50\% de todos os cânceres diagnosticados nos Estados Unidos de acordo com a organização mundial da saúde ${ }^{1-3}$. Os cânceres de pele são primariamente classificados como não melanoma e melanoma (Figura 1). O melanoma representa uma pequena porcentagem dos cânceres de pele diagnosticados anualmente (cerca de 3\%), porém é responsável por grande parte das mortes causadas por tumores de pele, chegando a $65 \%$ ao ano $^{4-6}$. A incidência do melanoma continua aumentando progressivamente, com aumento aproximado de 33\% nos homens e 26\% nas mulheres no período de 2002 a $2006^{7}$ e com cerca de 90.000 casos novos e 10.000 mortes nos Estados Unidos em 2017, de acordo com as estatísticas da American Cancer Society.

Os principais fatores de risco relacionados ao paciente são fototipo de pele, história pessoal e familiar de melanoma, presença de múltiplos

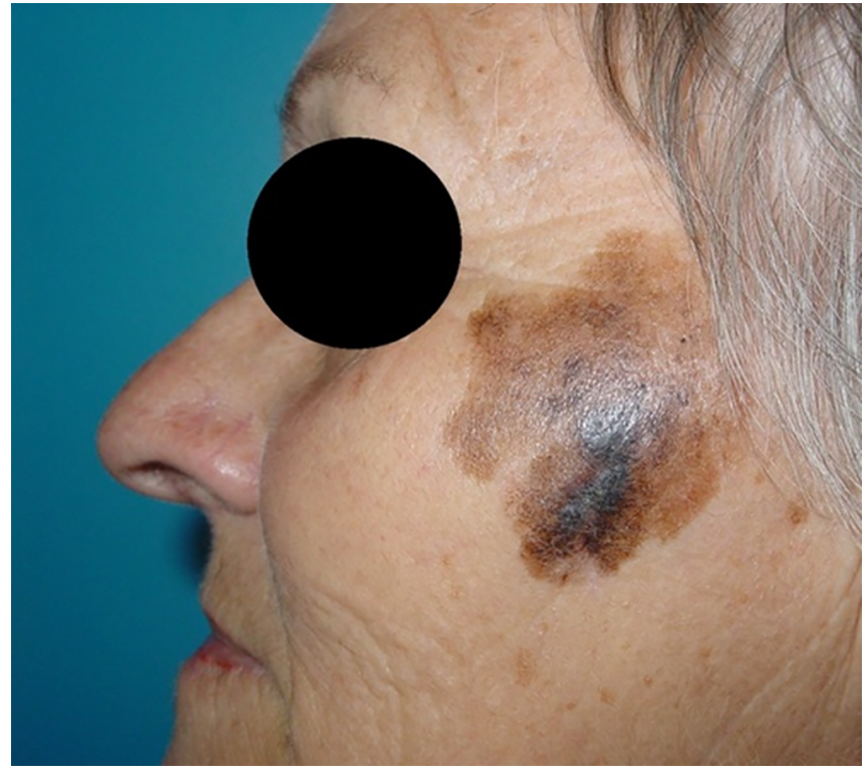

Figura 1. Lentigo maligno melanoma, um subtipo de melanoma, na face à esquerda de uma paciente com fototipo I.

nevos atípicos ou displásicos e fatores genéticos. Além desses, fatores ambientais, como exposição solar intensa ou esporádica, causando queimaduras com bolhas, e bronzeamento artificial com UVB, tem importante papel no desenvolvimento do melanoma ${ }^{8-19}$. Infelizmente muitos pacientes são

1 - Universidade Federal de São Paulo, Disciplina de Cirurgia Plástica, São Paulo, SP, Brasil. 
diagnosticados em estádio avançado, ou ainda, vivenciam progressão de doença, a despeito dos tratamentos instituídos. O melanoma evolui a partir de diversas lesões precursoras bem definidas, antes que se torne invasivo e metastático ${ }^{20,21}$. Diversos estudos analisaram os fatores preditivos de prognóstico relacionados ao melanoma, destacando-se, em somatória às características próprias do paciente, fatores relacionados ao tumor, como espessura de Breslow, presença de ulceração e índice mitótico, que foram identificados como os três fatores independentes mais importantes na análise dos desfechos dos pacientes ${ }^{22-34}$.

Portanto o conhecimento da epidemiologia e dos fatores de risco do melanoma cutâneo é de interesse para todos aqueles que estudam e trabalham com melanoma, incluindo cirurgiões, dermatologistas, oncologistas e médicos da atenção primária, para o desenvolvimento de novas campanhas de prevenção, ampliação do conhecimento existente e publicação de dados para atingir os profissionais que ainda não possuem familiaridade com a doença ${ }^{35}$.

Numerosos estudos têm investigado as características dos pacientes com melanoma e seus fatores prognósticos, porém, registros da América Latina e Brasil continuam escassos ${ }^{36}$. Desta forma, através deste estudo, procuramos entender melhor o perfil epidemiológico e patológico dos pacientes portadores de melanoma atendidos no Brasil e, assim, melhorar as estratégias do cuidado em nosso meio.

\section{MÉTODOS}

Estudo retrospectivo de dados epidemiológicos coletados do Setor de Tumores Cutâneos da Disciplina de Cirurgia Plástica do Hospital Universitário da Universidade Federal de São Paulo (UNIFESP). O estudo foi aprovado pelo Comitê de Ética e Pesquisa da UNIFESP sob o número 0986/11. Foi realizada análise dos registros hospitalares e ambulatoriais de 184 pacientes portadores de melanoma cutâneo atendidos no Serviço, de janeiro de 2005 a dezembro de 2010, com base em um protocolo que continha informações sobre sexo, cor, idade, profissão, exposição solar, características do tumor, localização da lesão, características histológicas, estadiamento e seguimento dos mesmos até o momento da finalização deste trabalho. Quanto à localização das lesões, dividimos em macrorregiões: cabeça e pescoço, tronco, membros superiores, membros inferiores ou de localização desconhecida.

Os dados coletados foram submetidos à análise estatística, em que foram utilizados testes não paramétricos. O nível de rejeição da hipótese de nulidade foi fixado em $5 \%$, considerando-se significante valor de $p=0,05$. Os resultados foram comparados a estudos epidemiológicos nacionais e internacionais.

\section{RESULTADOS}

Dos 184 pacientes, 103 (66\%) eram do sexo feminino e 81 (44\%) do sexo masculino. Quanto à cor da pele, 130 (70,6\%) foram classificados como brancos e 51 (27,7\%) como não brancos (pardos, negros e indígenas). Três (1,6\%) pacientes não tiveram informações sobre a cor da pele. A média de idade dos pacientes analisados foi de 58,7 anos no momento do diagnóstico. Dos pacientes analisados, $49(26,6 \%)$ trabalhavam expostos ao sol, 133 $(72,2 \%)$ trabalhavam sem exposição solar, e dois $(1,2 \%)$ não informaram sobre suas profissões.

Quanto aos aspectos histológicos, 116(63,1\%) apresentavam ao menos uma mitose por campo (índice mitótico), 42 (22,8\%) tinham índice mitótico igual a zero e, em 26 (14,1\%), não tivemos acesso ao exame histopatológico. Dos pacientes analisados, 125 (67,9\%) não apresentaram metástases linfonodais, 58 (31,5\%) apresentaram acometimento linfonodal; em um (0,6\%) paciente não temos esta informação.

A análise não mostrou diferença significante entreossexos, com $X^{2}$ calculadoiguala 0,93 ( $\left.p=0,6086\right)$. Quando analisamos a região acometida em relação 
ao sexo, encontramos diferenças estatisticamente significantes. O acometimento de cabeça, pescoço e tronco foi mais comum em homens e o de membros superiores e inferiores foi mais comum e, mulheres: $X^{2}$ calculado $=11,12(p=0,0111)$ (Tabela 1$)$.

Tabela 1. Distribuição de sexo para as regiões acometidas.

\begin{tabular}{lcccc}
\hline \multirow{2}{*}{ Região } & \multicolumn{2}{c}{ Feminino } & \multicolumn{2}{c}{ Masculino } \\
\cline { 2 - 5 } & $\mathrm{N}$ & $\%$ & $\mathrm{~N}$ & $\%$ \\
\hline Cabeça e pescoço & 17 & 17,0 & 23 & 29,1 \\
Tronco & 24 & 24,0 & 29 & 36,7 \\
Membros superiores & 17 & 17,0 & 9 & 11,3 \\
Membros inferiores & 42 & 42,0 & 18 & 22,9 \\
Total & 100 & 100 & 79 & 100 \\
\hline
\end{tabular}

Ao compararmos a exposição com a região acometida, os resultados sugerem uma relação entre a profissão com exposição solar e a ocorrência de melanoma em cabeça e pescoço, evidenciando o papel da exposição solar na gênese dessa neoplasia: $X^{2}$ calculado $=8,821(p=0,0318)$ (Tabela 2). Foram excluídos da análise sete pacientes (3,8\%) sem informações para fazer a comparação.

Tabela 2. Distribuição do fator de risco exposição dentro das regiões acometidas.

\begin{tabular}{|c|c|c|c|c|}
\hline \multirow{2}{*}{ Região } & \multicolumn{2}{|c|}{ Exposição } & \multirow{2}{*}{ Total } & \multirow{2}{*}{$\%$ de Sim } \\
\hline & Sim & Não & & \\
\hline Cabeça e pescoço & 14 & 26 & 40 & 35,0 \\
\hline Tronco & 18 & 34 & 52 & 34,6 \\
\hline Membros superiores & 9 & 17 & 26 & 34,6 \\
\hline Membros inferiores & 8 & 51 & 59 & 13,6 \\
\hline Total & 49 & 128 & 177 & 27,6 \\
\hline
\end{tabular}

Quando comparamos a incidência do fator de risco queimadura com a região acometida, a porcentagem de queimadura solar da cabeça e pescoço foi significantemente maior do que nas demais regiões e, ainda, a presença de queimaduras relacionadas a pacientes com melanoma em membros inferiores foi menor: $X^{2}$ calculado $=12,59$
( $p=0,0056)$ (Tabela 3). Foram excluídos da a análise 15 pacientes $(8,2 \%)$ sem informações para fazer a comparação.

Tabela 3. Distribuição do fator de risco queimadura solar dentro das regiões acometidas.

\begin{tabular}{lcccc}
\hline \multirow{2}{*}{ Região } & \multicolumn{3}{c}{ Queimadura } & \\
& \multicolumn{2}{c}{ Solar } & Total & \% de Sim \\
\cline { 2 - 3 } & Sim & Não & & \\
\hline Cabeça e pescoço & 20 & 19 & 39 & 51,2 \\
Tronco & 20 & 31 & 53 & 37,7 \\
Membros superiores & 9 & 17 & 24 & 37,5 \\
Membros inferiores & 9 & 44 & 53 & 16,9 \\
Total & 58 & 111 & 169 & 34 \\
\hline
\end{tabular}

Notamos que quanto mais cefálica a região maior é a taxa de pacientes dentro dela que apresentam queimaduras como fator de risco, evidenciando a baixa influência desse fator de risco na gênese de melanomas em regiões mais baixas e menos expostas. Ao compararmos a presença do fator de risco "exposição" com os sexos não obtivemos diferença estatisticamente significante: $X^{2}$ calculado $=1,35(p=0,2438)$ (Tabela 4). Foram excluídos da análise dois pacientes $(1,2 \%)$ que não tinham informação sobre exposição.

Tabela 4. Distribuição do fator de risco exposição dentro dos sexos.

\begin{tabular}{lcccc}
\hline \multirow{2}{*}{ Sexo } & \multicolumn{2}{c}{ Exposição } & \multirow{2}{*}{ Total } & \% de Sim \\
\cline { 2 - 3 } & Sim & Não & & \\
\hline Masculino & 25 & 55 & 80 & 31,2 \\
Feminino & 24 & 78 & 102 & 23,5 \\
Total & 49 & 133 & 182 & 30,2 \\
\hline
\end{tabular}

Ao compararmos o índice mitótico do melanoma com o acometimento linfonodal durante o seguimento, a análise mostrou associação significante entre a presença de uma ou mais mitoses e a ocorrência de metástase linfonodal, aumentando em 3,2 vezes o risco de metástases: $X^{2}$ calculado =8,71 ( $p=0,0032)$ (Tabela 5). 
Tabela 5. Distribuição de acometimento linfonodal e presença de mitoses.

\begin{tabular}{lcccc}
\hline \multirow{2}{*}{ Mitoses } & \multicolumn{2}{c}{ Linfonodo } & \multirow{2}{*}{ Total } & \% de Positivo \\
\cline { 2 - 4 } & Positivo & Negativo & & \\
\hline Presente & 42 & 74 & 116 & 36,2 \\
Ausente & 5 & 37 & 42 & 11,9 \\
Total & 47 & 111 & 125 & 37,6 \\
\hline
\end{tabular}

A relação entre a espessura de Breslow, obtida através do estudo anatomopatológico e o acometimento linfonodal, durante o seguimento, mostrou associação significante: $X^{2}$ calculado $=38,56(p<0,0001)$ (Tabela 6).

Tabela 6. Distribuição do acometimento linfonodal dentro das faixas de valor de Breslow.

\begin{tabular}{|c|c|c|c|c|}
\hline \multirow{2}{*}{ Breslow } & \multicolumn{2}{|c|}{ Linfonodo } & \multirow{2}{*}{ Total } & \multirow{2}{*}{$\%$ de Positivo } \\
\hline & Positivo & Negativo & & \\
\hline In situ (0) & 0 & 17 & 17 & 0,0 \\
\hline$<1$ & 4 & 35 & 39 & 10,2 \\
\hline 1,01 a 2 & 8 & 24 & 32 & 25,0 \\
\hline 2,01 a 4 & 5 & 20 & 25 & 25,0 \\
\hline$>4$ & 32 & 22 & 54 & 59,2 \\
\hline Total & 58 & 126 & 184 & 31,5 \\
\hline
\end{tabular}

\section{DISCUSSÃO}

Importante observar que a coleta dos dados foi prejudicada pela deficiência de registros da instituição, assim como costuma ocorrer no restante do Brasil ${ }^{36}$. Notamos que houve uma prevalência de mulheres em relação aos homens, situação comumente descrita para todas as doenças de pele. As razões pelas quais existe essa disparidade permanece desconhecida, mas provavelmente é multifatorial, incluindo diferenças nas camadas da pele e sua fisiologia, hormônios sexuais, idade, etnia, estilo de vida, ocupação entre outros ${ }^{37-39}$. Assim como outros dados encontrados na literatura, não houve diferença significativa entre os sexos dentro das faixas etárias mais acometidas ${ }^{40-43}$. Quanto à idade ao diagnóstico, notamos maior incidência nos pacientes acima de 50 anos.
Ao analisarmos os fatores de risco notoriamente conhecidos para o desenvolvimento de melanoma vimos que $70,6 \%$ dos pacientes eram considerados brancos, dos quais 49,4\% tinham pele tipo I ou II e 27,1\% tinham cabelos e olhos claros, evidenciando uma predisposição desses pacientes a ter melanoma pelo seu fenótipo. O fator de risco mais prevalente nos pacientes foi pele tipo I ou II, seguido da queimadura solar com bolhas durante a vida. Ao compararmos as regiões acometidas com a história de queimaduras solares vimos que regiões mais expostas, como cabeça e pescoço e tronco, estão mais relacionadas com pacientes que apresentaram queimaduras comparados aos pacientes que não apresentaram. Como descrito por outros autores ${ }^{20,44-46}$, as regiões mais acometidas foram cabeça e pescoço e tronco, somando $50,1 \%$.

Identificamos que os homens são mais afetados em regiões como cabeça, pescoço e tronco, o que pode ser explicado por uma maior exposição solar no trabalho dos homens em relação às mulheres. Além disso, conseguimos observar diferença estatística quando comparamos a presença de exposição solar com a área acometida, evidenciando o papel da exposição em melanomas de cabeça e pescoço e tronco e sua baixa relação com regiões menos expostas ${ }^{43}$.

A informação mais importante que o estudo detectou em relação ao prognóstico dos pacientes surgiu através da análise da relação entre a presença de mitoses e a espessura de Breslow com o acometimento metastático de linfonodos. Verificamos que a presença de apenas uma mitose por campo na avaliação histopatológica está associada à metástase linfonodal, levando o paciente ao estádio III, com mal prognóstico. Estes pacientes têm indicação de tratamento adjuvante pela disseminação da doença, dado que está de acordo com diversos estudos internacionais ${ }^{44-47}$. Da mesma forma, ao compararmos as espessuras 
de Breslow com o acometimento linfonodal notamos que quanto maior o Breslow, maior é o risco de metástase. Nosso estudo vem reafirmar essa informação importante, publicada na revisão internacional sobre fatores prognósticos e estadiamento do melanoma cutâneo, realizado pela American Joint Comittee on Cancer ${ }^{48,49}$.

Em nosso estudo, encontramos diversas informações que reafirmam a literatura existente até hoje sobre fatores de risco mais incidentes para o desenvolvimento do melanoma, como pele e olhos claros, a exposição ao sol e as queimaduras solares, colocando os fatores de risco em duas categorias: fatores fenotípicos e não passíveis de prevenção, e fatores externos, como a radiação solar, que é passível de prevenção ${ }^{49,50}$.

Com isto, estimulamos medidas de prevenção do melanoma, como campanhas de diminuição à exposição solar e incentivo ao uso de filtros solares, assim como, a identificação de fatores de mal prognóstico, a fim de obter melhor acompanhamento dos pacientes.

\title{
A B S T R A C T
}

\begin{abstract}
Objective: to evaluate the characteristics of the patients with cutaneous melanoma treated at the São Paulo Hospital UNIFESP. Methods: we conducted a retrospective study of 184 cases of cutaneous melanoma. We analyzed information on gender, age, tumor characteristics, histological characteristics and staging. Results: mean age at diagnosis was 58.7 years, with homogeneous age distribution between genders and predominance in white individuals (70.6\%). There was a predominance of trunk involvement in men (36.7\%) and lower limbs in women (42\%). Sun exposure, with sunburns, was more common among males (31.2\%) than among females (23.5\%). There was an approximately three-fold increase in lymph node involvement when the mitotic index rose from zero (11.9\%) to one or more mitosis per field (36.2\%). In addition, the greater the Breslow thickness, the greater the lymph node involvement and poor the outcomes: $10.2 \%$ when less than $1 \mathrm{~mm}$ and $59.2 \%$ when greater than $4 \mathrm{~mm}$. Conclusion: the characteristics of patients with cutaneous melanoma treated at Hospital São Paulo are similar to those found in the literature.
\end{abstract}

Keywords: Melanoma. Skin Neoplasms. Mitosis. Risk Factors. Melanomalepidemiology.

\section{REFERÊNCIAS}

1. World Health Organization. Cancer Research UK. World cancer factsheet. London: World Health Organ; 2014.

2. Mariotto $A B$, Yabroff $K R$, Shao $Y$, Feuer EJ, Brown $\mathrm{ML}$. Projections of the cost of cancer care in the United States: 2010-2020. J Natl Cancer Inst. 2011;103(2):117-28.

3. Carpenter WR, Yeh WS, Wobker SE, Godley PA. Getting cancer prevalence right: using state cancer registry data to estimate cancer survivors. Cancer Causes Control. 2011;22(5):765-73.

4. Dzwierzynski WW. Managing malignant melanoma. Plast Reconstr Surg. 2013;132(3):446e-60e. Erratum in. Plast Reconstr Surg. 2014;133(3):762.

5. Siegel R, Ward E, Brawley O, Jemal A. Cancer Statistics, 2011: the impact of eliminating socioeconomic and racial disparities on premature cancer deaths. CA Cancer J Clin. 2011;61(4):212-36.
6. Pavri SN, Clune J, Ariyan S, Narayan D. Malignant melanoma: beyond the basics. Plast Reconstr Surg. 2016;138(2):330e-40e.

7. Jemal A, Saraiya M, Patel P, Cherala SS, BarnholtzSloan J, Kim J, et al. Recent trends in cutaneous melanoma incidence and death rates in the United States, 1992-2006. J Am Acad Dermatol. 2011;65(5 Suppl 1):S17-25. e1-3.

8. Naeyaert JM, Brochez L. Clinical practice. Dysplastic nevi. N Engl J Med. 2003;349(23):2233-40.

9. Rigel DS, Rivers JK, Kopf AW, Friedman RJ, Vinokur AF, Heilman ER, et al. Dysplastic nevi. Markers for increased risk for melanoma. Cancer. 1989;63(2):386-9.

10. Evans RD, Kopf AW, Lew RA, Rigel DS, Bart RS, Friedman RJ, et al. Risk factors for the development of malignant melanoma--I: Review of case-control studies. J Dermatol Surg Oncol. 1988;14(4):393-408. 
11. Góralska A, Blaszczyk J. Characteristics of risk factors for development of melanocytic naevi and melanoma in patients presented to a dermatologist to assess melanocytic lesions. Przegl Dermatol. 2013;100(1):86-95.

12. Williams ML, Sagebiel RW. Melanoma risk factors and atypical moles. West J Med. 1994;160(4):343-50.

13. Ivry GB, Ogle CA, Shim EK. Role of sun exposure in melanoma. Dermatol Surg. 2006;32(4):481-92. Erratum in: Dermatol Surg. 2006;32(6):preceding 773.

14. Colantonio S, Bracken MB, Beecker J. The association of indoor tanning and melanoma in adults: systematic review and meta-analysis. J Am Acad Dermatol. 2014;70(5):847-57. e1-18.

15. Lazovich $D$, Vogel Rl, Berwick $M$, Weinstock MA, Anderson KE, Warshaw EM. Indoor tanning and risk of melanoma: a case-control study in a highly exposed population. Cancer Epidemiol Biomarkers Prev. 2010;19(6):1557-68.

16. Elliott F, Suppa M, Chan M, Leake S, Karpavicius B, Haynes $S$, et al. Relationship between sunbed use and melanoma risk in a large case-control study in the United Kingdom. Int J Cancer. 2012;130(12):3011-3.

17. Zhang M, Qureshi AA, Geller AC, Frazier L, Hunter DJ, Han J. Use of tanning beds and incidence of skin cancer. J Clin Oncol. 2012;30(14):1588-93.

18. Bentzen J, Krarup AF, Castberg IM, Jensen PD, Philip A. Determinants of sunbed use in a population of Danish adolescents. Eur J Cancer Prev. 2013;22(2):126-30.

19. Stapleton JL, Hillhouse J, Turrisi R, Robinson JK, Baker K, Manne SL, et al. Erythema and ultraviolet indoor tanning: findings from a diary study. Transl Behav Med. 2013;3(1):10-6.

20. Gordon D, Gillgren P, Eloranta S, Olsson H, Gordon $\mathrm{M}$, Hansson J, et al. Time trends in incidence of cutaneous melanoma by detailed anatomical location and patterns of ultraviolet radiation exposure: a retrospective population-based study. Melanoma Res. 2015;25(4):348-56.

21. Shain AH, Yeh I, Kovalyshyn I, Sriharan A, Talevich E, Gagnon A, et al. The genetic evolution of melanoma from precursor lesions. N Engl J Med. 2015;373(20):1926-36.

22. Rozeman EA, Dekker TJA, Haanen JBAG, Blank CU. Advanced melanoma: current treatment options, biomarkers, and future perspectives. Am J Clin Dermatol. 2018;19(3):303-17.

23. Shain $A H$, Bastian $B C$. From melanocytes to melanomas. Nat Rev Cancer. 2016;16(6):345-58.

24. Svedman FC, Pillas D, Taylor A, Kaur M, Linder R, Hansson J. Stage-specific survival and recurrence in patients with cutaneous malignant melanoma in Europe - a systematic review of the literature. Clin Epidemiol. 2016;8:109-22.

25. Balch CM, Gershenwald JE, Soong SJ, Thompson JF, Atkins MB, Byrd DR, et al. Final version of 2009 AJCC melanoma staging and classification. J Clin Oncol. 2009;27(36):6199-206.

26. National Comprehensive Cancer Network. NCCN Clinical practice guidelines in oncology. Melanoma. Fort Washington (PA): NCCN; 2016.

27. Maurichi A, Miceli R, Camerini T, Mariani L, Patuzzo $R$, Ruggeri $R$, et al. Prediction of survival in patients with thin melanoma: results from a multi-institution study. J Clin Oncol. 2014;32(23):2479-85.

28. Ericksson $H$, Frohm-Nilsson $M$, Järås J, KanterLewensohn L, Kjellman P, Månsson-Brahme $E$, et al. Prognostic factors in localized invasive primary cutaneous malignant melanoma: results of a large population-based study. Br J Dermatol. 2015;172(1):175-86.

29. Ransohoff KJ, Jaju PD, Tang JY, Carbone M, Leachman S, Sarin KY. Familial skin cancer syndromes: increased melanoma risk. J Am Acad Dermatol. 2016;74(3):423-34.

30. Nikolaou $V$, Stratigos AJ. Emerging trends in the epidemiology of melanoma. $\mathrm{Br} J$ Dermatol. 2014;170(1):11-9.

31. Aoude LG, Wadt KA, Pritchard AL, Hayward NK. Genetics of familial melanoma: 20 years after CDKN2A. Pigment Cell Melanoma Res. 2015;28(2):148-60.

32. Edge SB, Carducci M, Byrd DR, eds. AJCC Cancer Staging Manual. 7th ed. New York: Springer-Verlag; 2009.

33. Balch CM, Gershenwald JE, Soong SJ, Thompson JF, Ding S, Byrd DR, et al. Multivariate analysis of prognostic factors among 2,313 patients with stage III melanoma: comparison of nodal micrometastases versus macrometastases. J Clin Oncol. 2010;28(14):2452-9. 
34. Thompson JF, Soong SJ, Balch CM, Gershenwald JE, Ding S, Coit DG, et al. Prognostic significance of mitotic rate in localized primary cutaneous melanoma: an analysis of patients in the multi-institutional American Joint Committee on Cancer melanoma staging database. J Clin Oncol. 2011;29(16):2199-205. Erratum in: J Clin Oncol. 2011;29(21):2949.

35. MacKie RM, Hauschild A, Eggermont AM. Epidemiology of invasive cutaneous melanoma. Ann Oncol. 2009;20 Suppl 6: vi1-7.

36. Vazquez Vde L, Silva TB, Vieira Mde A, de Oliveira AT, Lisboa MV, de Andrade DA, et al. Melanoma characteristics in Brazil: demographics, treatment, and survival analysis. BMC Res Notes. 2015;8:4.

37. Chen W, Mempel M, Traidl-Hofmann C, Al Khusaei S, Ring J. Gender aspects in skin diseases. J Eur Acad Dermatol Venereol. 2010;24(12):1378-85.

38. Pérez-Gómez B, Aragonés N, Gustavsson P, Lope $V$, López-Abente $\mathrm{G}$, Pollán $\mathrm{M}$. Do sex and site matter? Different age distribution in melanoma of the trunk among Swedish men and women. Br J Dermatol. 2008;158(4):766-72.

39. Lowe GC, Saavedra A, Reed KB, Velazquez Al, Dronca RS, Markovic SN, et al. Increasing incidence of melanoma among middle-aged adults: an epidemiologic study in Olmsted County, Minnesota. Mayo Clin Proc. 2014; 89(1):52-9.

40. Reed KB, Brewer JD, Lohse CM, Bringe KE, Pruitt $C N$, Gibson LE. Increasing incidence of melanoma among young adults: an epidemiological study in Olmsted County, Minnesota. Mayo Clin Proc. 2012;87(4):328-34.

41. Siegel R, Naishadham D, Jemal A. Cancer statistics, 2013. CA Cancer J Clin. 2013;63(1):11-30.

42. Erdmann F, Lortet-Tieulent J, Schüz J, Zeeb H, Greinert R, Breitbart EW, et al. International trends in the incidence of malignant melanoma 19532008--are recent generations at higher or lower risk? Int J Cancer. 2013;132(2):385-400.

43. Ivry GB, Ogle CA, Shim EK. Role of sun exposure in melanoma. Dermatol Surg. 2006;32(4):481-92.

44. Colantonio S, Bracken MB, Beecker J. The association of indoor tanning and melanoma in adults: systematic review and meta-analysis. J Am Acad Dermatol. 2014;70(5):847-57.e1-18.
45. Kirkland EB, Zitelli JA. Mitotic rate for thin melanomas: should a single mitotic figure warrant a sentinel lymph node biopsy? J Dermatol Surg. 2014;40(9):937-45.

46. Gershenwald JE, Scolyer RA, Hess KR, Sondak VK, Long GV, Ross MI, Lazar AJ, Faries MB, Kirkwood JM, McArthur GA, Haydu LE, Eggermont AMM, Flaherty KT, Balch CM, Thompson JF; for members of the American Joint Committee on Cancer Melanoma Expert Panel and the International Melanoma Database and Discovery Platform. Melanoma Staging: Evidence-based changes in the American Joint Committee on Cancer eighth edition cancer staging manual. CA Cancer J Clin. 2017;67(6):472-92.

47. Wat $H$, Senthilsenvan A, Salopek TG. A retrospective, multicenter analysis of the predictive value of mitotic rate for sentinel lymph node (SLN) positivity in thin melanomas. J Am Acad Dermatol. 2016;74(1):94-101.

48. Kibrité A, Milot $H$, Douville $P$, Gagné ÉJ, Labonté $S$, Friede J, et al. Predictive factors for sentinel lymph nodes and non-sentinel lymph nodes metastatic involvement: a database study of 1,041 melanoma patients. Am J Surg. 2016;211(1):89-94.

49. Chhabra G, Ndiaye MA, Garcia-Peterson LM, Ahmad N. Melanoma chemoprevention: current status and future prospects. Photochem Photobiol. 2017;93(4):975-89.

50. Green AC, Williams GM, Logan V, Strutton GM. Reduced melanoma after regular sunscreen use: randomized trial follow-up. J Clin Oncol. 2011;29(3):257-63.

Recebido em: 08/01/2018

Aceito para publicação em: 17/05/2018

Conflito de interesse: nenhum.

Fonte de financiamento: nenhuma

Endereço para correspondência: Tácito Ferreira

E-mail: tacito.ferreira@hotmail.com dra.afo@gmail.com

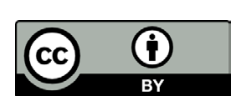

